\title{
Rheumatoid Arthritis is Not Associated with Increased Inpatient Mortality in Patients Admitted for Acute Coronary Syndrome
}

\author{
Ehizogie Edigin ${ }^{1}$, Hafeez Shaka ${ }^{1}$, Precious Eseaton ${ }^{2}$, Shakeel Jamal ${ }^{3}$, Asim Kichloo ${ }^{4}$, Pius E. Ojemolon \\ , Iriagbonse Asemota ${ }^{1}$, Emmanuel Akuna ${ }^{1}$, Augustine Manadan 6 \\ 1. Internal Medicine, John H. Stroger, Jr. Hospital of Cook County, Chicago, USA 2. Internal Medicine, University of \\ Benin, Benin City, NGA 3. Internal Medicine, Central Michigan University College of Medicine, Saginaw, USA 4. \\ Internal Medicine, Central Michigan University, Saginaw, USA 5. Anatomical Sciences, St. George's University, St. \\ George's, GRD 6. Rheumatology, John H. Stroger, Jr. Hospital of Cook County, Chicago, USA
}

Corresponding author: Ehizogie Edigin, ediginehizogie@yahoo.com

\begin{abstract}
Objectives: This study aims to compare the outcomes of patients admitted primarily for acute coronary syndrome (ACS) with and without a secondary diagnosis of rheumatoid arthritis (RA).
\end{abstract}

Methods: Data were abstracted from the National Inpatient Sample (NIS) 2016 and 2017 Database. The NIS was searched for hospitalizations of adult patients with ACS as principal diagnoses, with and without RA as a secondary diagnosis. The primary outcome was inpatient mortality. Secondary outcomes were hospitalization characteristics and cardiovascular therapies. Multivariate logistic and linear regression analysis were used accordingly to adjust for confounders.

Results: There were over 71 million discharges included in the combined 2016 and 2017 NIS database. Out of 1.3 million patients with ACS, 22,615 (1.7\%) had RA. RA group was older ( 70.4 vs 66.8 years, $\mathrm{P}<0.001)$ as compared to the non-RA group, and had more females (63.7\% vs $37.7 \%, \mathrm{P}<0.0001)$. Patients with RA had a $16 \%$ reduced risk of in-hospital mortality: odds ratio (OR) $0.84,95 \%$ confidence interval (CI) (0.72-0.99), $\mathrm{P}=0.034$; less odds of undergoing intra-aortic balloon pump (IABP): OR 0.78, $95 \% \mathrm{CI}(0.64-0.95), \mathrm{P}=0.015$; and 0.18 days shorter hospital length of stay (LOS): $95 \% \mathrm{CI}(0.32-0.05), \mathrm{P}=0.009$. However, odds of undergoing percutaneous coronary intervention with drug-eluting stent (PCI DES) at OR 1.14, 95\% CI (1.071.23), $\mathrm{P}<0.0001$ was significantly higher in the RA group compared to ACS without RA.

Conclusions: Patients admitted for ACS with co-existing RA had lower adjusted inpatient mortality, less odds of undergoing IABP, shorter adjusted LOS, and greater adjusted odds of undergoing PCI DES compared to those without RA.

Received 07/10/2020 Review began 07/10/2020 Review ended 08/03/2020 Published 08/17/2020

(c) Copyright 2020 Edigin et al. This is an open access article distributed under the terms of the Creative Commons Attribution License CC-BY 4.0., which permits unrestricted use, distribution, and reproduction in any medium, provided the original author and source are credited.
Categories: Cardiology, Internal Medicine, Rheumatology

Keywords: rheumatoid arthritis, cardiovascular diseases, rheumatic diseases, disease modifying anti-rheumatic drugs, hospitalization, mortality

\section{Introduction}

Rheumatoid arthritis (RA) is a debilitating, chronic autoimmune disorder that has both articular and extraarticular manifestations with marked systemic inflammation [1,2]. Acute coronary syndrome (ACS) refers to any group of clinical symptoms consistent with acute myocardial ischemia and includes ST-segment elevation myocardial infarction (STEMI), non-ST-segment elevation myocardial infarction (NSTEMI), and unstable angina (UA) [3].

Cardiovascular (CV) diseases are the leading cause of mortality and morbidity among patients with RA [4-6]. These patients are at increased risk compared to patients without coexisting RA due to chronic systemic inflammation [7-11]. The effect of this increased CV risk on outcomes of ACS in RA has been scarcely studied with different results $[10,12,13]$. Some studies concluded that ACS outcomes are worse in RA, while others showed decreased mortality of ACS in RA after adjusting for traditional CV risk factors like hypertension, diabetes mellitus (DM), tobacco, and family history of CV disease [14]. Though it is well established in the literature that RA increases the risk of CV events, there remains a scarcity of national population-based research on outcomes of ACS in RA patients. This study aims to add to the body of knowledge and fill the knowledge gap of the uncertainty of the impact of RA on outcomes of patients admitted for ACS.

\section{Materials And Methods}

\section{Data source}

We conducted a retrospective cross-sectional study of hospitalizations in 2016 and 2017 with a principal 
diagnosis of ACS with and without a secondary diagnosis of RA in acute-care hospitals across the United States. Hospitalizations were selected from the NIS database. The NIS was created and is maintained by the Agency for Healthcare Research and Quality and is the largest publicly available all-payer inpatient database in the United States. It was designed as a stratified probability sample to be representative of all nonfederal acute-care hospitals nationwide. Hospitals are stratified according to ownership, urban or rural location, geographic region, bed size, and teaching status. A 20\% probability sample of all hospitals within each stratum is then collected. All discharges from these hospitals are recorded and then weighted to ensure that they are nationally representative. The 2016 and 2017 NIS sampling frame includes data from 47 statewide data organizations (46 states plus the District of Columbia) covering more than $97 \%$ of the U.S. population. 30 discharge diagnoses for each hospitalization can be recorded using the International Classification of Diseases, Tenth Revision, Clinical Modification (ICD-10) in NIS 2016, and as many as 40 discharge diagnoses in NIS 2017 database. In the NIS, diagnoses are divided into two separate categories: principal diagnosis and secondary diagnoses. A principal diagnosis was the main ICD-10 code for the hospitalization. Secondary diagnoses were any ICD-10 code other than the principal diagnosis. There is no reliable mechanism to distinguish secondary diagnoses that antedated the hospital admission from those that had onset during the index admission. Since all patient data in NIS are de-identified and publicly available, institutional review board approval was not sought.

\section{Inclusion criteria and study variables}

The study population consisted of all inpatient hospitalizations recorded in the NIS 2016 and 2017. Study variables included age, gender, race, hospital characteristics, medical comorbidities, primary and secondary outcomes (outlined below). We used the following ICD-10 codes to identify principal/secondary diagnoses: ACS all I21.0, I21.1, I21.2, I21.3, 121.4, I20.0 codes, and RA all M05 and M06 codes (see appendix). We studied baseline characteristics and outcomes for ACS hospitalization with and without RA.

\section{Outcomes}

The primary outcome was inpatient mortality. Rates of percutaneous transluminal coronary angioplasty (PTCA)/coronary atherectomy, percutaneous coronary intervention (PCI) with drug-eluting stent (PCI DES), PCI with bare-metal stent (PCI BMS), intra-aortic balloon pump (IABP), percutaneous external assist device (PEAD), intracoronary artery thrombolytic infusion, coronary artery bypass surgery (CABG), mean hospital length of stay (LOS) and mean total hospitalization charges were compared as secondary outcomes of interest.

\section{Statistical analysis}

Analyses were performed using STATA version 16 (StataCorp, Texas, USA). A univariate logistic regression analysis using all variables and comorbidities in Table 1 was used to calculate unadjusted odds ratios (ORs) for the primary outcome. All variables with $\mathrm{P}$-values $<0.1$ were included in a multivariate logistic regression model. Univariate association of variables and comorbidities with the primary outcome, highlighting the variables included in the multivariable logistic regression model, are displayed in Table 2 . P-values $<0.05$ were considered significant in the multivariate analysis. Confounders were selected from literature review. Charlson comorbidity index, which is recorded in the NIS database, was used to adjust for comorbidity burden. Multivariate logistic and linear regression model, with all variables and comorbidities in Table 1, were used to adjust for confounders for the secondary outcomes.

\section{Results}

There were over 71 million discharges included in the combined 2016 and 2017 NIS database. Out of 1,319,464 hospitalizations for ACS, 22,615 (1.7\%) had RA, while 1,296,849 (98.3\%) did not have RA. Characteristics of ACS hospitalizations with and without co-existing RA are displayed in Table 1. RA group was older (70.4 vs 66.8 years, $\mathrm{P}<0.0001)$, and had more females (63.7\% vs $37.7 \%, \mathrm{P}<0.0001)$.

\begin{tabular}{|c|c|c|c|}
\hline & \multicolumn{3}{|l|}{ ACS $(n=1,319,464)$} \\
\hline & Without RA ( $n=1,296,849)$ & With RA $(n=22,615)$ & P-value \\
\hline Mean Age (years) & 66.8 & 70.4 & $<0.0001$ \\
\hline Female & $37.7 \%$ & $63.7 \%$ & $<0.0001$ \\
\hline Race & & & $<0.0001$ \\
\hline White & $73.7 \%$ & $76.8 \%$ & Reference \\
\hline Black & $11.4 \%$ & $10.8 \%$ & 0.054 \\
\hline Hispanic & $8.6 \%$ & $7.5 \%$ & 0.005 \\
\hline Asians & $2.8 \%$ & $1.9 \%$ & $<0.0001$ \\
\hline
\end{tabular}




\section{Cureus}

$\begin{array}{ll}\text { Native Americans } & 0.55 \% \\ \text { Others } & 3.0 \%\end{array}$

$0.55 \%$

$0.55 \%$
$3.0 \%$

Charlson comorbidity index

\section{0}

1

2

$\geq 3$

Hospital bed size

Small

Medium

Large

Hospital teaching status

Nonteaching

Teaching

Hospital location

Rural

Urban

Expected primary payer

\section{Medicare}

Medicaid

Private

Self-pay

Median household income (quartile)

$$
\begin{aligned}
& 1^{\text {st }}\left(0-25^{\text {th }}\right) \\
& 2^{\text {nd }}\left(26^{\text {th }}-50^{\text {th }}\right) \\
& 3^{\text {rd }}\left(51^{\text {st }}-75^{\text {th }}\right)
\end{aligned}
$$$$
4^{\text {th }}\left(76^{\text {th }}-100^{\text {th }}\right)
$$

Hospital region

Northeast

Midwest

South

West

Dyslipidemia

Old MI

Old PCl

Old CABG

Old pacemaker

A. Fib/flutter

COPD

$0.55 \%$

$24.4 \%$

$24.7 \%$

$50.3 \%$

$16.6 \%$

$30.5 \%$

$52.9 \%$

$33.6 \%$

$66.5 \%$

$7.8 \%$

$92.2 \%$

$9.8 \%$

$26.6 \%$

$4.7 \%$

$30.9 \%$

$27.5 \%$

$23.4 \%$

$18.3 \%$
$0.60 \%$

$2.4 \%$

0.847

0.017

$<0.0001$

$18.7 \%$

$18.7 \%$

$34.5 \%$

$65.5 \%$

$9.6 \%$

$90.4 \%$

$74.3 \%$

$6.2 \%$

$17.8 \%$

$1.7 \%$

$30.5 \%$

$27.9 \%$

$22.9 \%$

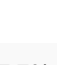

$17.7 \%$

$25.3 \%$

$39.1 \%$

$17.9 \%$

$64.3 \%$

$16.6 \%$

$1.8 \%$

$8.7 \%$

$3.5 \%$

$22.7 \%$

$26.0 \%$
0.0086

0.0172

0.7893

0.0009

0.0632

$<0.0001$

0.6092

0.1962

$<0.0001$

$<0.0001$

0.7483

0.0001

$<0.0001$ 


\section{Cureus}

\begin{tabular}{|llll|} 
Carotid artery disease & $2.3 \%$ & $2.5 \%$ & 0.2685 \\
Old stroke & $1.1 \%$ & $1.2 \%$ & 0.5856 \\
Hypertension & $47.9 \%$ & $48.6 \%$ & 0.3366 \\
Peripheral vessel disease & $6.8 \%$ & $7.5 \%$ & 0.0532 \\
Hypothyroidism & $11.7 \%$ & $19.4 \%$ & $<0.0001$ \\
DM type 1 \& 2 & $40.0 \%$ & $35.8 \%$ & $<0.0001$ \\
Obesity & $18.2 \%$ & $16.4 \%$ & 0.0024 \\
CHF & $33.4 \%$ & $36.8 \%$ & $<0.0001$ \\
CKD & $23.5 \%$ & $24.7 \%$ & 0.0454 \\
\hline Liver disease & $3.4 \%$ & $3.1 \%$ & 0.2586 \\
Electrolyte derangement & $21.4 \%$ & $23.9 \%$ & $<0.0001$ \\
\hline Maintenance hemodialysis & $3.2 \%$ & $2.2 \%$ & 0.0003 \\
\hline O2 dependence & $2.4 \%$ & $4.0 \%$ & $<0.0001$ \\
\hline Smoking & $27.2 \%$ & $31.8 \%$ & $<0.0001$ \\
\hline Anemia & $22.6 \%$ & $29.9 \%$ & $<0.0001$ \\
\hline
\end{tabular}

\section{TABLE 1: Baseline characteristics of ACS hospitalizations with and without RA}

MI: myocardial infarction; PCI: percutaneous coronary intervention; CABG: coronary artery bypass graft; COPD: chronic obstructive pulmonary disease; DM: diabetes mellitus; CHF: chronic congestive heart failure; CKD: chronic kidney disease; O2: oxygen; A. Fib/flutter: Atrial fibrillation/flutter; median household income: median household income for patient's ZIP code

Univariate association of the baseline characteristics with the primary outcome is shown in Table 2.

\begin{tabular}{|c|c|c|}
\hline Baseline variables & Odds Ratio & P-value \\
\hline Age $^{\star}$ & 1.04 & $<0.0001$ \\
\hline Female gender* & 1.17 & $<0.0001$ \\
\hline \multicolumn{3}{|l|}{ Race $^{\star}$} \\
\hline White & Reference & Reference \\
\hline Black & 0.81 & $<0.0001$ \\
\hline Hispanic & 0.91 & 0.013 \\
\hline Asians & 1.21 & $<0.0001$ \\
\hline Native Americans & 0.83 & 0.174 \\
\hline Others & 1.07 & 0.152 \\
\hline Charleston comorbidity index & 1.21 & $<0.0001$ \\
\hline \multicolumn{3}{|l|}{ Hospital bed size } \\
\hline Small & Reference & Reference \\
\hline Medium & 1.09 & 0.002 \\
\hline Large & 1.14 & $<0.0001$ \\
\hline Hospital teaching status* & 1.09 & $<0.0001$ \\
\hline Hospital location & 1.04 & 0.186 \\
\hline Expected primary paye & & \\
\hline
\end{tabular}




\section{Cureus}

\begin{tabular}{|c|c|c|}
\hline Medicare & Reference & Reference \\
\hline Medicaid & 0.49 & $<0.0001$ \\
\hline Private & 0.39 & $<0.0001$ \\
\hline Self-pay & 0.60 & $<0.0001$ \\
\hline \multicolumn{3}{|c|}{ Median household income(quartile)* } \\
\hline $1^{\text {st }}\left(0-25^{\text {th }}\right)$ & Reference & Reference \\
\hline $2^{\text {nd }}\left(26^{\text {th }}-50^{\text {th }}\right)$ & 1.00 & 0.874 \\
\hline $3^{\text {rd }}\left(51^{\text {st }}-75^{\text {th }}\right)$ & 0.93 & 0.010 \\
\hline $4^{\text {th }}\left(76^{\text {th }}-100^{\text {th }}\right)$ & 1.01 & 0.713 \\
\hline \multicolumn{3}{|l|}{ Hospital region* ${ }^{\star}$} \\
\hline Northeast & Reference & Reference \\
\hline Midwest & 0.93 & 0.010 \\
\hline South & 0.98 & 0.385 \\
\hline West & 1.02 & 0.542 \\
\hline Dyslipidemia* ${ }^{*}$ & 0.44 & $<0.0001$ \\
\hline Old $\mathrm{Ml}^{\star}$ & 0.78 & $<0.0001$ \\
\hline Old PCl${ }^{\star}$ & 0.82 & 0.011 \\
\hline Old CABG & 1.05 & 0.106 \\
\hline Old pacemaker* & 1.17 & 0.002 \\
\hline A. Fib/flutter* & 1.85 & $<0.0001$ \\
\hline COPD* $^{*}$ & 1.26 & $<0.0001$ \\
\hline Carotid artery disease & 1.00 & 0.976 \\
\hline Old stroke* & 1.49 & $<0.0001$ \\
\hline Hypertension* ${ }^{*}$ & 0.48 & $<0.0001$ \\
\hline Peripheral vessel disease ${ }^{*}$ & 1.42 & $<0.0001$ \\
\hline Hypothyroidism & 1.09 & 0.002 \\
\hline DM type 1 \& 2 & 1.02 & 0.248 \\
\hline Obesity* & 0.57 & $<0.0001$ \\
\hline $\mathrm{CHF}^{*}$ & 2.36 & $<0.0001$ \\
\hline $\mathrm{CKD}^{*}$ & 1.86 & $<0.0001$ \\
\hline Liver disease ${ }^{\star}$ & 7.07 & $<0.0001$ \\
\hline Electrolyte derangement ${ }^{\star}$ & 4.79 & $<0.0001$ \\
\hline Maintenance hemodialysis* & 1.83 & $<0.0001$ \\
\hline O2 dependence ${ }^{\star}$ & 1.36 & $<0.0001$ \\
\hline Smoking* & 0.83 & $<0.0001$ \\
\hline Anemia ${ }^{*}$ & 1.67 & $<0.0001$ \\
\hline
\end{tabular}

TABLE 2: Univariate association of baseline variables with inpatient mortality

MI: myocardial infarction; PCl: percutaneous coronary intervention; CABG: coronary artery bypass graft; COPD: chronic obstructive pulmonary disease; DM: diabetes mellitus; CHF: chronic congestive heart failure; CKD: chronic kidney disease; O2: oxygen; median household income: median 


\section{Cureus}

household income for patient's ZIP code

* variable included in the multivariable logistic regression model

60,750 adult ACS hospitalizations (4.6\%) resulted in inpatient mortality. 1,010 (4.5\%) of the deaths occurred in ACS hospitalizations with coexisting RA vs 59,740 (4.6\%) in those without co-existing RA ( $\mathrm{P}=0.6637$ ). After adjusting for comorbidities (see Table 2 ) in the multivariate logistic regression analysis, hospitalizations for ACS (STEMI, NSTEMI, and UA) with RA had a 16\% reduced risk of in-hospital mortality, OR: $0.8495 \%$ confidence interval (CI) (0.72- 0.99), $\mathrm{P}=0.034$, compared to those without RA. Outcomes of ACS hospitalizations with and without co-existing RA are displayed in Table 3.

\begin{tabular}{|c|c|c|c|c|}
\hline & ACS with RA (n=22,615) & ACS without RA $(n=1,296,849)$ & Adjusted OR & p-value \\
\hline & $\%$ & $\%$ & $(95 \% \mathrm{Cl})$ & \\
\hline \multicolumn{5}{|l|}{ Primary outcome } \\
\hline In-hospital mortality & 4.5 & 4.6 & $0.84(0.72-0.99)$ & $0.034^{\star}$ \\
\hline \multicolumn{5}{|l|}{ Secondary outcomes } \\
\hline PTCA & 4.3 & 5.1 & $0.94(0.81-1.10)$ & 0.451 \\
\hline PCI DES & 35.4 & 40.5 & $1.14(1.07-1.23)$ & $<0.0001^{*}$ \\
\hline PCI BMS & 7.1 & 7.9 & $1.01(0.90-1.14)$ & 0.884 \\
\hline IABP & 2.8 & 4.0 & $0.78(0.64-0.95)$ & $0.015^{\star}$ \\
\hline PEAD & 0.88 & 1.0 & $1.07(0.77-1.48)$ & 0.697 \\
\hline Thrombolytics & 0.24 & 0.29 & $1.07(0.56-2.05)$ & 0.836 \\
\hline \multirow[t]{2}{*}{ CABG } & 6.9 & 8.1 & $0.95(0.83-1.09)$ & 0.473 \\
\hline & & & \multicolumn{2}{|c|}{ Adjusted mean difference } \\
\hline LOS, mean days & 4.6 & 4.4 & $-0.18(-\{0.32-0.05\}$ & $0.009^{*}$ \\
\hline Total charge, mean, USD & 85,273 & 91,889 & $-888(\{-3845\}-2070)$ & 0.556 \\
\hline
\end{tabular}

\section{TABLE 3: Clinical outcomes of ACS hospitalizations with and without RA}

PTCA: percutaneous transluminal coronary angioplasty; PCI DES: percutaneous coronary intervention with drug-eluting stent; PCI BMS: percutaneous coronary intervention with bare-metal stent; IABP: intra-aortic balloon pump; PEAD: percutaneous external assist devices; thrombolytics: intracoronary artery thrombolytic infusion; CABG: coronary artery bypass graft; OR: odds ratio; CI: confidence interval; USD: United States dollars

* statistically significant

Hospitalizations for ACS with RA had less odds of undergoing IABP, OR: 0.78, 95\% CI (0.64- 0.95), P=0.015, and 0.18 days shorter adjusted mean LOS $95 \%$ CI (0.32-0.05), $\mathrm{P}=0.009$ compared to those without RA. However, the adjusted odds of undergoing percutaneous coronary intervention with drug-eluting stent (PCI DES), OR: 1.14, 95\% CI (1.07-1.23), P<0.0001 was significantly higher in ACS with RA group compared to ACS without RA.

\section{Discussion}

The main findings of our study are: 1) patients admitted for ACS with co-existing RA had less adjusted inpatient mortality and LOS compared to those without RA. 2) ACS hospitalizations with co-existing RA had greater adjusted odds of undergoing PCI DES, but less odds of undergoing IABP placement compared to those without RA. IABP is used for refractory cardiogenic shock, which is a known complication of acute myocardial infarction [15].

In our study, the RA group had less traditional CV risk factors such as dyslipidemia, DM, obesity, and maintenance hemodialysis. However, the RA group had more smokers, CHF, CKD, and atrial fibrillation/flutter. We adjusted for all these differences in the multivariate logistic model. 
A large population-based study comparing five-year mortality of incident RA cases compared to the general population showed improvement in mortality of 2001-2006 cohort compared to the 1996-2000 cohort [16]. The improved outcome of RA patients after the year 2000 in this study was attributed to improved management of RA with disease-modifying anti-rheumatic drugs (DMARDS) and resultant lower underlying inflammation [16]. This is similar to a 15-year prospective cohort Dutch study which showed increased mortality (particularly for CV diseases) in RA patients compared to the general population; however, the mortality tended to decrease over time [17]. RA patients have 1.5-2.0 times increased risk of developing coronary artery disease (CAD) compared to the general population, which is similar to DM $[6,18,10]$. An interplay between traditional CV risk factors, chronic inflammation, and RA treatment (such as high dose corticosteroids) has been implicated in the pathogenesis of the increased CV risk in RA [19].

Most large population-based studies on RA are about the mortality risk of RA compared to the general population, and on the increased risk of CV disorders in RA. There is a scarcity of studies comparing outcomes of ACS in RA patients to ACS patients without RA. We found three national population-based studies on outcomes of ACS in RA patients with rather contrasting results.

The first study was done in Taiwan. This study showed increased in-hospital mortality of RA patients with acute myocardial infarction (MI) [20]. This may be due to the difference in availability and utilization of DMARDS for RA in the U.S. population compared to the Taiwanese population. This is supported by studies from developing countries, which showed higher mortality rates in RA relative to developed countries like the US [21].

The second study was a nation-wide population-based Swedish cohort study, which found that seven and 30-day short-term mortality after ACS was worse in RA cohort vs general population [12]. This is an older study with cohort follow-up period between January 2007 to December 2010. The results from this Swedish population study may not be generalizable to the U.S. population due to the difference in both health care systems and the sociodemographic difference between both populations. Information on known CAD risk factors such as body mass index (BMI) and smoking was not available, therefore, were not adjusted for during the analysis of this study.

Our results are more aligned with the third study, a U.S.-based population study using NIS 2002-2016 database, which showed that RA is associated with lower inpatient mortality in acute MI [22]. It was hypothesized that the use of immunotherapy had a modulatory and possibly a protective effect in the RA cohort. This study used older NIS databases and combined ICD- 9 and 10 codes, which can be problematic. In our study, we used the two most recent releases of the NIS databases from the ICD-10 era.

Our study has several strengths. First, we utilized data from the most recent hospitalization database available at the US population level (NIS 2016 and 2017). Second, we had a large sample size, which would give the study a high power. Third, we exclusively used the latest ICD-10 codes, which helped to further characterize the cohort and outline comorbidities that could have been confounders in prior studies.

There are some limitations to our study. First, NIS database analysis is subject to biases of retrospective studies. Second, there is a possibility of error associated with coding, as the NIS is an administrative database that uses ICD-10 codes to identify diseases and hospitalization events. Third, most of the ICD-10 billing codes fail to grade disease severity. Thus, we cannot determine if underlying RA disease severity/active disease status affected the outcome of ACS. Fourth, NIS reports data on hospitalizations, rather than individual patients. Therefore, patients hospitalized multiple times cannot be discerned. Fifth, data on specific DMARDs used by patients, rate of use, medication adherence, as well as laboratory, and radiologic data which could indicate inflammatory activity and disease severity are lacking in the NIS database. Lastly, differences in treatment modalities (PCI DES and IABP) may be confounded by indication, rather than the presence or absence of RA. However, we hope the large sample size and improved uniformity in diagnostic and therapeutic coding would compensate for these deficiencies. More research is needed to understand the mechanism by which co-existing RA imparts outcomes of patients admitted for ACS.

\section{Conclusions}

Patients admitted for ACS with co-existing RA had lower adjusted inpatient mortality, less odds of undergoing IABP, shorter adjusted LOS, and greater adjusted odds of undergoing PCI DES compared to those without RA. Although RA increases the risk of developing ACS, RA does not negatively impact outcomes of ACS hospitalizations based on this large U.S. national database.

\section{Appendices}




\section{Cureus}

ACS

RA

I21.0, I21.1, I21.2, I21.3, 121.4, 120.0

Procedure

codes

PTCA

PCI BMS

PCI DES

IABP

PEAD

Intracoronary

artery

thrombolytic

infusion

CABG

Comorbidities

M05, M06 027045Z, 0273476, $027347 Z$

$5 A 02210,5 A 02110$

3E07017, 3E07317

02703ZZ, 02704ZZ, 02713ZZ, 02714ZZ, 02723ZZ, 02724ZZ, 02733ZZ, 02734ZZ

02703D6, 02703DZ, 02704D6, 02704DZ, 02703E6, 02703EZ, 02704E6, 02704EZ, 02703F6, 02703FZ, 02704F6, 02704FZ, 02703G6, 02703GZ, 02704G6, 02704GZ, 02713D6, 02713DZ, 02714D6, 02714DZ, 02713E6, 02713EZ, 02714E6, 02714EZ, 02713F6, 02713FZ, 02714F6, 02714FZ, 02713G6, 02713GZ, 02714G6, 02714GZ, 02723D6, 02723DZ, 02724D6, 02724DZ, 02723E6, 02723EZ, 02724E6, 02724E6, 02724EZ, 02723F6, 02723FZ, 02724F6, 02724FZ, 02723G6, 02723GZ, 02724G6, 02724GZ, 02733D6, 02733DZ, 02734D6, 02734DZ, 02733E6, 02733EZ, 02734E6, 02734EZ, 02733F6, 02733FZ, 02733FZ, 02734F6, 02733G6, 02733GZ, 02734G6, 02734GZ

0270346, 027034Z, 0270446, 027044Z, 0270356, 027035Z, 0270456, 027045Z, 0270366, 027036Z, 0270466, 027046Z, 0270376, 027037Z, 0270476, 027047Z, 0271346, 027134Z, 0271446, 027144Z, 0271356, 027135Z, 0271456, 027145Z, 0271366, 027136Z, 0270376, 0271466, 027146Z, 0271376, 027137Z, 0271476, 027147Z, 0272346, 027234Z, 0272446, 027244Z, 0272356, 027235Z, 0272456, 027245Z, 0272366, 027236Z, 027246Z, 0272376, 027237Z, 0272476, 027035Z, 027247Z, 0273346, 027334Z, 0273446, 027344Z, 0273356, 027335Z, 0273456, 027345Z, 0273366, 027336Z, 0273466, 027346Z, 0273376, 027337Z,

02HAORJ, 02HA3RJ, 02HA4RJ, 5A02116, 5A0211D, 5A02216, 5A0221D, 02HA3RZ, 5A02216

0210093, 0210098, 0210099, 021009C, 021009F, 021009W, 02100A3, 02100A8, 02100A9, 02100AC, 02100AF, 02100AW, 0211093, 0211098, 0211099, 021109C, 021109F, 021109W, 02110A3, 02110A8, 02110A9, 02110AC, 02110AF, 02110A, 0212093, 0212098, 0212099, 021209C, 021209F, 021209W, 02120A3, 02120A8, 02120A9, 02120AC, 02120AF, 02120AW, 0213093, 0213098, 0213099, 021309C, 021309F, 021309W, 02130A3, 02130A8, 02130A9, 02130AC, 02130AF, 02130AW

\begin{tabular}{|c|c|}
\hline Dyslipidemia & E78 \\
\hline Old MI & 1252 \\
\hline Old PCl & Z9861 \\
\hline Old CABG & Z951 \\
\hline Old pacemaker & Z950 \\
\hline $\begin{array}{l}\text { Atrial } \\
\text { fibrillation/flutter }\end{array}$ & 148 \\
\hline $\begin{array}{l}\text { Chronic } \\
\text { obstructive } \\
\text { pulmonary } \\
\text { disease }\end{array}$ & $\mathrm{J} 41, \mathrm{~J} 42, \mathrm{~J} 43, \mathrm{~J} 44$ \\
\hline $\begin{array}{l}\text { Carotid artery } \\
\text { disease }\end{array}$ & 1652 \\
\hline Old stroke & 163 \\
\hline Hypertension & 110 \\
\hline $\begin{array}{l}\text { Peripheral } \\
\text { vascular } \\
\text { disease }\end{array}$ & 1739 \\
\hline Hypothyroidism & E03 \\
\hline $\begin{array}{l}\text { Diabetes } \\
\text { mellitus type } 1 \text { \& } \\
2\end{array}$ & E10, E11 \\
\hline
\end{tabular}




\section{Cureus}

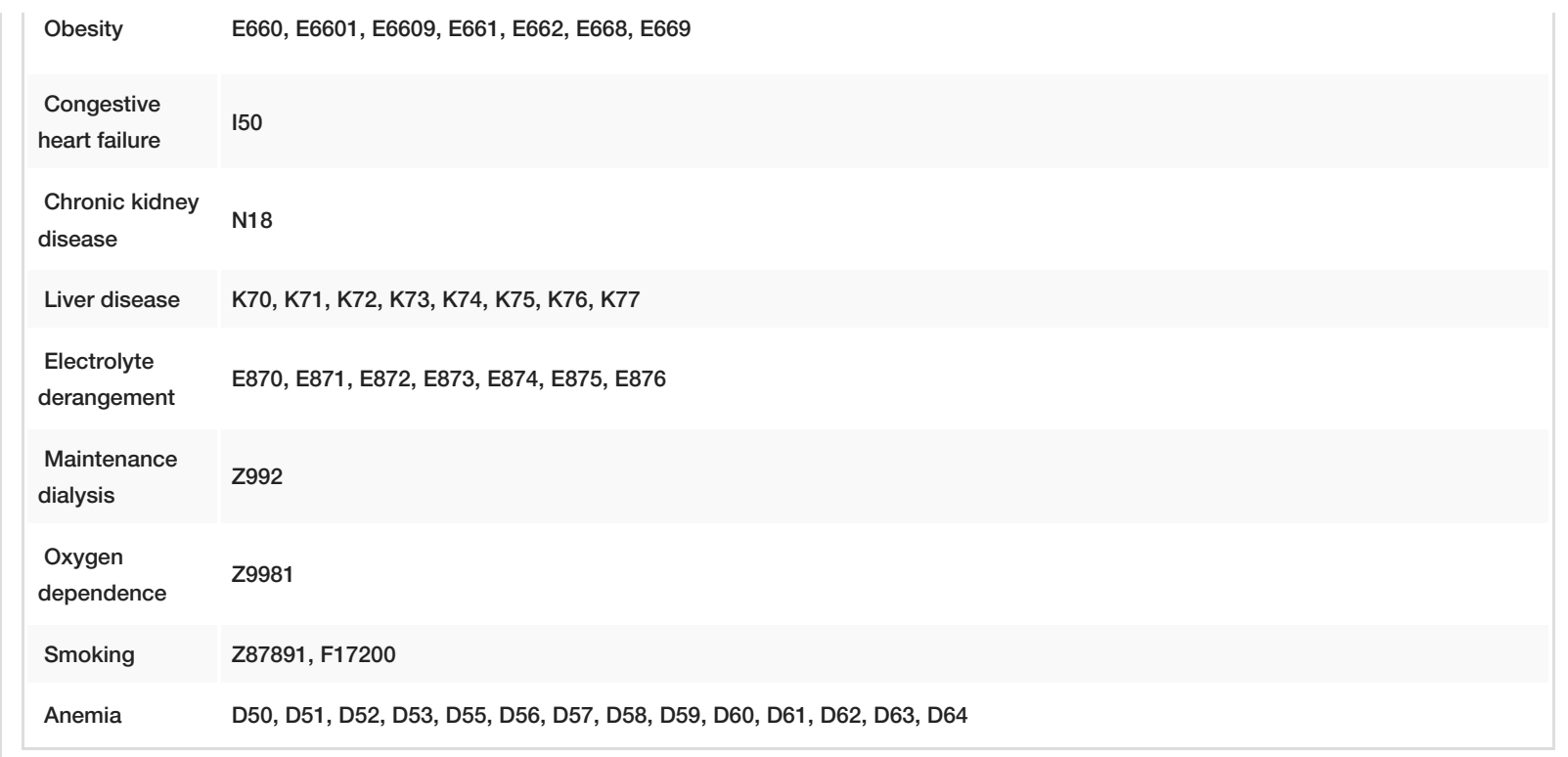

TABLE 4: Used ICD-10 codes

\section{Additional Information}

\section{Disclosures}

Human subjects: Consent was obtained by all participants in this study. N/A issued approval N/A. Since all patient data in the National Inpatient Sample (NIS) database are de-identified and publicly available, institutional review board approval was not sought. Animal subjects: All authors have confirmed that this study did not involve animal subjects or tissue. Conflicts of interest: In compliance with the ICMJE uniform disclosure form, all authors declare the following: Payment/services info: All authors have declared that no financial support was received from any organization for the submitted work. Financial relationships: All authors have declared that they have no financial relationships at present or within the previous three years with any organizations that might have an interest in the submitted work. Other relationships: All authors have declared that there are no other relationships or activities that could appear to have influenced the submitted work.

\section{References}

1. Scott DL, Wolfe F, Huizinga TWJ: Rheumatoid arthritis. Lancet. 2010, 376:1094-108. 10.1016/S01406736(10)60826-4

2. McInnes IB, O’Dell JR: State-of-the-art: rheumatoid arthritis. Ann Rheum Dis. 2010, 69:1898-906. 10.1136/ard.2010.134684

3. Kumar A, Cannon CP: Acute coronary syndromes: diagnosis and management, part I . Mayo Clin Proc. 2009, 84:917-38.

4. Meune C, Touzé E, Trinquart L, Allanore T: Trends in cardiovascular mortality in patients with rheumatoid arthritis over 50 years: a systematic review and meta-analysis of cohort studies. Rheumatology. 2009, 48:1309-13. 10.1093/rheumatology/kep252

5. Wallberg-Jonsson S, Ohman ML, Dahlqvist SR: Cardiovascular morbidity and mortality in patients with seropositive rheumatoid arthritis in Northern Sweden. J Rheumatol. 1997, 24:445-51.

6. Solomon DH, Goodson NJ, Katz JN, et al.: Patterns of cardiovascular risk in rheumatoid arthritis . Ann Rheum Dis. 2006, 65:1608-12. 10.1136/ard.2005.050377

7. Solomon DH, Karlson EW, Rimm EB, et al.: Cardiovascular morbidity and mortality in women diagnosed with rheumatoid arthritis. Circulation. 2003, 107:1303-7. 10.1161/01.cir.0000054612.26458.b2

8. Solomon DH, Avorn J, Katz JN, Weinblatt ME, Setoguchi S, Levin R, Schneeweiss S: Immunosuppressive medications and hospitalization for cardiovascular events in patients with rheumatoid arthritis. Arthritis Rheum. 2006, 54:3790-8. 10.1002/art.22255

9. Myasoedova E, Chandran A, Ilhan B, Major BT, Michet CJ, Matteson EL, Crowson ES: The role of rheumatoid arthritis (RA) flare and cumulative burden of RA severity in the risk of cardiovascular disease. Ann Rheum Dis. 2016, 75:560-5. 10.1136/annrheumdis-2014-206411

10. Peters MJL, van Halm VP, Voskuyl AE, et al.: Does rheumatoid arthritis equal diabetes mellitus as an independent risk factor for cardiovascular disease? a prospective study. Arthritis Rheum. 2009, 61:1571-9. 10.1002/art.24836

11. Zhang J, Chen L, Delzell E, et al.: The association between inflammatory markers, serum lipids and the risk of cardiovascular events in patients with rheumatoid arthritis. Ann Rheum Dis. 2014, 73:1301-8. 10.1136/annrheumdis-2013-204715

12. Mantel Ä, Holmqvist M, Jernberg T, Wållberg-Jonsson S, Askling J: Rheumatoid arthritis is associated with a 


\section{Cureus}

more severe presentation of acute coronary syndrome and worse short-term outcome. Eur Heart J. 2015, 36:3413-22. 10.1093/eurheartj/ehv461

13. Wolfe F, Mitchell DM, Sibley JT, et al.: The mortality of rheumatoid arthritis . Arthritis Rheum. 1994, 37:48194. 10.1002/art.1780370408

14. Solomon DH, Curhan GC, Rimm EB, Cannuscio CC, Karlson EW: Cardiovascular risk factors in women with and without rheumatoid arthritis. Arthritis Rheum. 2004, 50:3444-9. 10.1002/art.20636

15. Thomaz PG, Moura LA, Muramoto G, Assad RS: Intra-aortic balloon pump in cardiogenic shock: state of the art. Rev Col Bras Cir. 2017, 44:102-6. 10.1590/0100-69912017001006

16. Lacaille D, Avina-Zubieta JA, Sayre EC, Abrahamowicz M: Improvement in five-year mortality in rheumatoid arthritis compared to the general population - closing the mortality gap. Ann Rheum Dis. 2017, 76:1057-63. 10.1136/annrheumdis-2016-209562

17. van den Hoek J, Boshuizen HC, Roorda LD, Tijhuis GJ, Nurmohamed MT, van den Bos GAM, Dekker J: Mortality in patients with rheumatoid arthritis: a 15-year prospective cohort study . Rheumatol Int. 2017, 37:487-93. 10.1007/s00296-016-3638-5

18. Maradit-Kremers H, Crowson CS, Nicola PJ, Ballman KV, Roger VL, Jacobsen SJ, Gabriel SE: Increased unrecognized coronary heart disease and sudden deaths in rheumatoid arthritis: a population-based cohort study. Arthritis Rheum. 2005, 52:402-11. 10.1002/art.20853

19. Crowson CS, Liao KP, Davis JM, et al.: Rheumatoid arthritis and cardiovascular disease . Am Heart J. 2013, 166:622-8. 10.1016/j.ahj.2013.07.010

20. Lai C-H, Hsieh C-Y, Barnado A, et al.: Outcomes of acute cardiovascular events in rheumatoid arthritis and systemic lupus erythematosus: a population-based study. Rheumatology. 2019, 59:1355-63. 10.1093/rheumatology/kez456

21. Meyer PW, Anderson R, Ker JA, Ally MTM: Rheumatoid arthritis and risk of cardiovascular disease . Cardiovasc J Afr. 2018, 29:317-21. 10.5830/CVJA-2018-018

22. Elbadawi A, Ahmed HH, Elgendy IY, et al.: Outcomes of acute myocardial infarction in patients with rheumatoid arthritis [IN PRESS]. Am J Med. 2020, 10.1016/j.amjmed.2020.02.039 